\title{
IMPROVING POWER SYSTEM PERFORMANCE USING DEMAND-SIDE MANAGEMENT WITHIN SMART GRIDS ENVIRONMENT
}

\author{
${ }^{1}$ Ahmed M. Azmy, ${ }^{2}$ A.Barakatand ${ }^{3}$,A.Abaza \\ ${ }^{1}$ Electrical Power and Machines Engineering Department, \\ Faculty of Engineering, Tanta, University, \\ Tanta, Egypt, (azmy.ahmed@hotmail.com, azmy@uni-duisburg.de) \\ ${ }^{2}$ Electrical Engineer, the Electrical Engineering Department, \\ Faculty of Engineering, KaferelShiekh University \\ KaferelShiekh, Egypt, (eng20067@yahoo.com) \\ ${ }^{3}$ Assisstant lecturer, The Electrical Engineering Department, \\ Faculty of Engineering, KaferelShiekh University \\ KaferelShiekh, Egypt, (abazaam_1428@yahoo.com)
}

\begin{abstract}
This paper introduces a method to improve the performance of power systems using demand side management to overcome the problems of increasing load demand. Defining suitable demand reduction levels can cause a significant enhancement for the entire network. Therefore, the sensitivity factors related to each load centre are defined to rank the load centres according to their priority. According to these factors, the best required load reduction can be obtained at each load centre. The recommended reduction level has to ensure overcoming the problems associated with the voltage profile and transmission lines overloading due to the increase in load demand. The IEEE 30 bus system is used to highlight the demand-side management strategy under different loading conditions. Some simulation results are introduced to show the importance of considering the sensitivity of load centres when defining the reduction levels at each load centre. The recommended demand side management is compared with the load shedding technique as an emergency action to avoid the problems of line violation and to keep the system stable and secure. According to the obtained results, considerable improvement is achieved in the voltage profile, the network efficiency and power loss without the need for the load shedding. These results encourage the implementation of the introduced technique considering sensitivity of load centres in smart grid environment.
\end{abstract}

Keywords: Demand side management, Dynamic pricing, Load shedding, Smart grid.

\section{Introduction}

The utilization of electric energy, which is a main support to industry development, has been increased significantly in the last years [1]. The load-demand increase causes some contingencies in transmission lines and can cause violation in line constraints [2]. The constraints violation affects the security of the entire power system. Even under normal operation, the power quality will be affected with this loading due to, e.g. the higher voltage drop[3], [4]. Conventionally, the power 
system components have to be upgraded according to the load increase. Alternatively, distributed generation and renewable energy sources, such as wind turbine and photovoltaic, can be used. This is normally accomplished based on extremely expensive plan study and may not be sufficient in some cases to match available generation with the demand [5], [6]. Otherwise, the load shedding is necessary to prevent any over loading on the network. However, this cannot be accepted from customers since the supply continuity represents a main requirement for them.

Recently, Demand Side Management "DSM" is applied as an important methodology to overcome the problems associated with load-demand increase. The DSM enhances the utilization of the transmission system by shifting loads to other periods [7]. Some residential loads can be shifted in time without bothering customers such as washing machines [1], [8] but this process has to be achieved by customer participation.

In [8], a control methodology to emerge DSM with distributed generation and distributed storage is introduced. An advanced system is developed in [9] using programmable appliances at sites of consumer to take intelligent decisions automatically according to real time demand data. In [10] on the other hand, the management is accomplished using cell phones, which display information and allow consumers to control electric appliances. In [7], a design of a smart home controller is proposed to provide efficient electricity management of domestic loads depending on forecasting the electric demand of consumers. In [4], a methodology is proposed to install capacitors and voltage regulators at distribution networks to control the voltage magnitude with the load increase.

These solutions need special arrangements to control the residential loads, which increases the cost considerably. In addition, the management can take place independently on customers, which can cause some problems and may be impractical. Furthermore, these methods do not differentiate between consumers from the side view of their effect on the network performance.

This paper investigates the utilization of DSM technique depending on the influence of different load centres on the network performance. The main idea is to differentiate between different loads according to the sensitivity of the network to their variation. Therefore, the effect of load variation on the network behaviour is deeply studied using the IEEE 30-bus system. The idea is to define the loads that have higher influence on power flow, losses, cost of generation and bus voltages. The effect of load reduction based on sensitivity factors is compared to the case of random load reduction to ensure the effectiveness of distinguishing different customers. The operation within smart grid will enable using bi-directional communication to evaluate system situation and send suitable reduction levels to customers depending on their sensitivity factors. In addition, smart meters can be used to display the energy price according to the response of each load centre.

\section{Problem Formulation}

The continuous increase of electricity utilization affects the performance of electrical networks [1]. It can be observed on the IEEE 30-bus system, for instance, that the load increase causes overpower flow on the lines and in certain situations, the maximum power limits are violated. The number of violated lines depends on the percentage increase of the load demand. Hence, it is possible to reduce or even eliminate the power limit violation by decreasing the load demand through the customers contribution. The participation of customers not only affects the magnitude of power flow, but also the quality, amount of generated power, cost of generation and bus voltages.

\section{Demand-Side Management Algorithm}

\section{a. System description}

The IEEE 30-bus system is chosen to investigate the DSM strategy. The possibility of participating different loads on the management process will be studied and the effect of this process on the performance of the network will be evaluated. Table (1) summarized the description of this network. 
Table 1, parameters of the IEEE 30-bus system

\begin{tabular}{|l|l|l|l|}
\hline Buses & 30 & Loads & 20 \\
\hline Generators & 6 & Branches & 41 \\
\hline Committed Gens & 6 & Transformers & 0 \\
& & & \\
\hline
\end{tabular}

The problem of overloaded lines can be solved by load reduction. The demand reduction can be performed randomly or according to the effect of each load on the performance of the entire network. In all cases, the load has to be reduced to the level that eliminates any violation in the transmission-lines limits. Any scenario of load reduction has to take into consideration the buses that have more effect on the network performance. Hence, the more sensitive buses have to be defined and taken into account since they can affect the system when respond to the signal for reducing their demand. These buses can be identified by the so-called coefficient of sensitivity (sensitivity factor).

\section{b. $\quad$ Sensitivity factor}

The sensitivity factor is used to define the more sensitive buses. The sensitivity analysis for the system (30 buses) can be determined by the following steps [11].

1.The value of a certain load is increased by a certain value $\Delta \mathrm{S}_{\mathrm{i}}$, while other loads are maintained at their original values.

2.The change in the power flowing in all transmission lines $\Delta \mathrm{S}_{\mathrm{TL}-\mathrm{ij}}$ is defined

3.The sensitivity factor for each bus $\mathrm{i}$ " $S F_{i}$ " is determined using the following equation:

$$
\mathrm{SF}_{\mathrm{i}}=\frac{\sum_{\substack{\mathrm{i}=1 \\ \mathrm{j} \neq \mathrm{i}}}^{\mathrm{n} \Delta \mathrm{S}_{\mathrm{TL}-\mathrm{ij}}}}{\Delta \mathrm{S}_{\mathrm{i}}}
$$

4.The buses are ranked according to their sensitivity factors from the highest value to the lowest value.

\section{c. Effect of considering sensitive load centres}

Due to the change in load demand, the generation cost, losses and bus voltages are changed. If the total reduction in load demand is requested from all load centres randomly or by the same percentage, the network performance will be improved. However, this improvement may not be enough to achieve the required operation of the network. On the other hand, considering the sensitivity factors of each load centre can attain better performance and satisfy system constraints when requesting the same total demand reduction. Fig (1) and Fig (2), respectively, show the network efficiency and losses with different loading reduction levels after satisfying all constraints. Two curves are given for the cases of considering and omitting the sensitivity factors when the load is reduced. 
The numbers on curves indicate the \% loading after reduction

The numbers on the columns refersto the percentages loadings after reduction

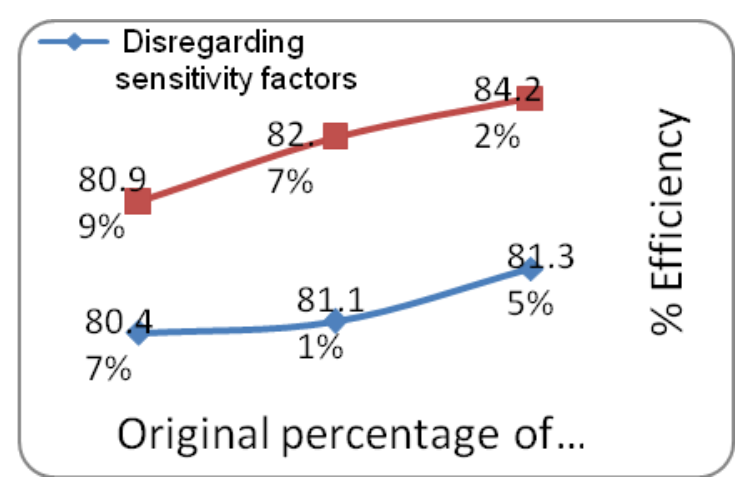

Fig (1) Network efficiency under different load reductions

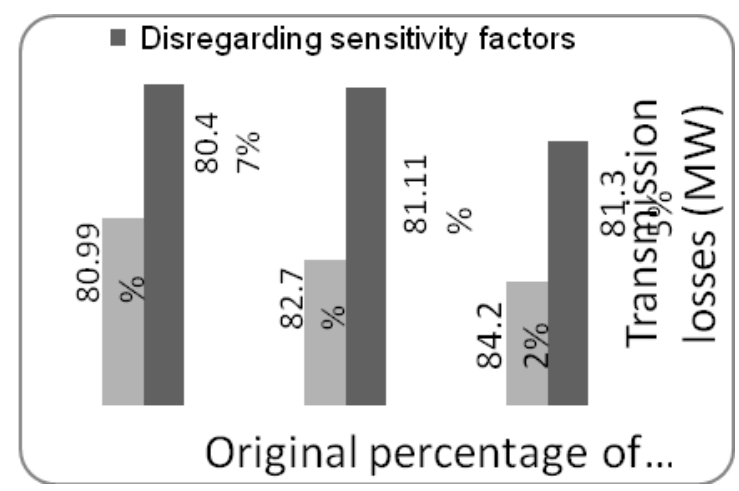

Fig (2) Transmission losses with different load reductions

These figures show that reducing load demands in the IEEE 30-bus network considering sensitivity factors gives better results considering both the network efficiency and the losses. The required percentage load reduction to eliminate the violation of the line constraints is lower when the sensitivity factors are considered. For $95 \%$ loading for instance, the load has to be reduced to $84.22 \%$ considering sensitivity factors, compared to $81.35 \%$ for random reduction.

Fig (3) and Fig (4) compare between the bus voltages of the IEEE 30-bus network considering and disregarding the sensitivity factors.

For the two figures, the load demand at load centres is reduced from $95 \%$ to $84.22 \%$ and from $85 \%$ to $80.99 \%$ respectively. Regarding the voltage levels, considering sensitivity factors gives better voltage profile for the same load reduction level. It is clear from the figures that the load reduction is more effective when sensitivity factors are considered. Furthermore, considering sensitivity factor results in satisfying the line constrains, while some line limits are violated when the reduction is carried out randomly. To reach the point where all constrains are satisfied, more reduction is required for random variation.

These figures show that reducing the load demand according to sensitivity factors is more effective regarding the network performance. This means that the signals indicating the required demand reduction to customers have to take the sensitivity analysis into account. Different reduction levels have to be defined and sent to customers according to the sensitivity factor of each load centre. This necessitates that the percentage load reduction level at each load centre must be defined online to solve the corresponding problem of violating line constraints due to the increase of load demand. This can be achieved by heuristic calculations, which are based on the value of sensitivity factor of each load centre. 


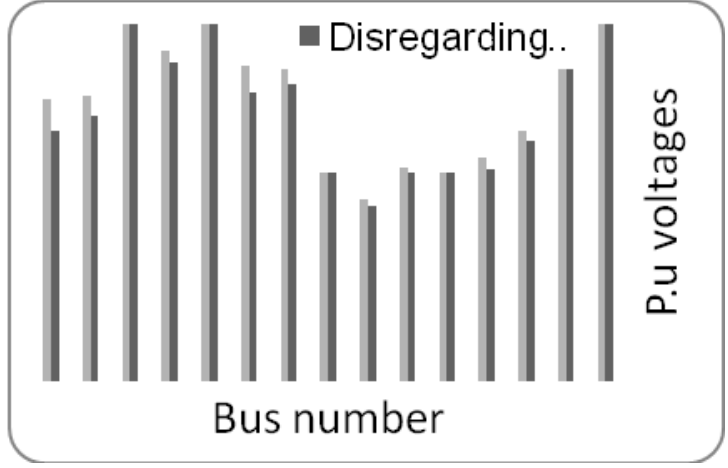

Fig (3-a) Buses from 1 to 15

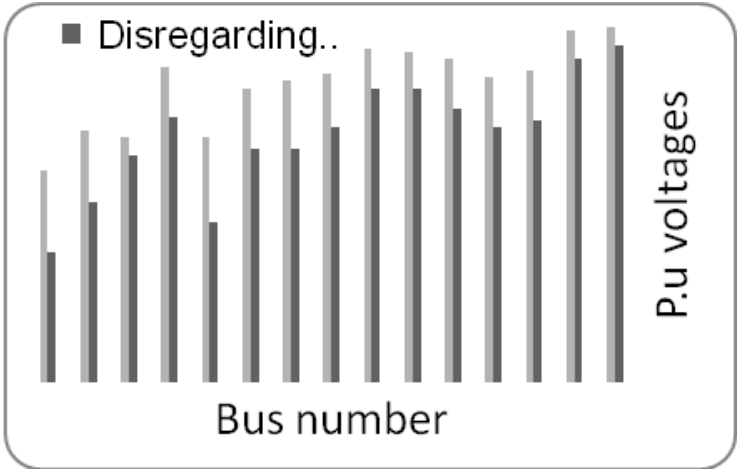

Fig (3-b) Buses from 16 to 30

Fig (3) Bus voltages when demand is reduced from $95 \%$ to $84.22 \%$

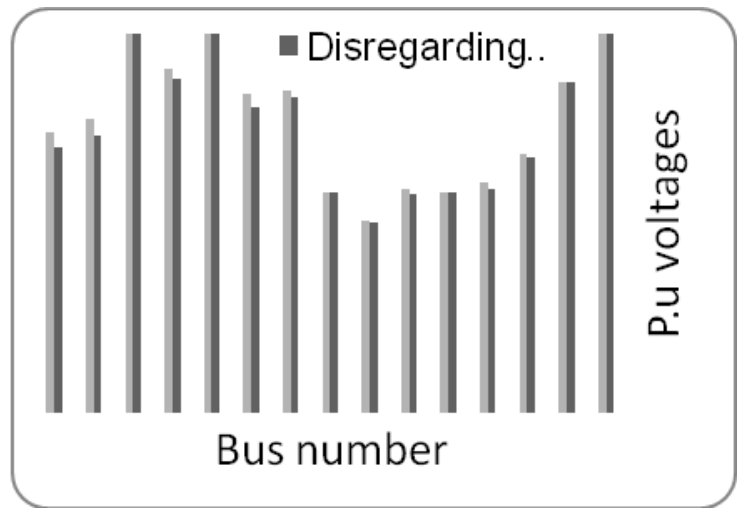

Fig (4-a) Buses from 1 to 15

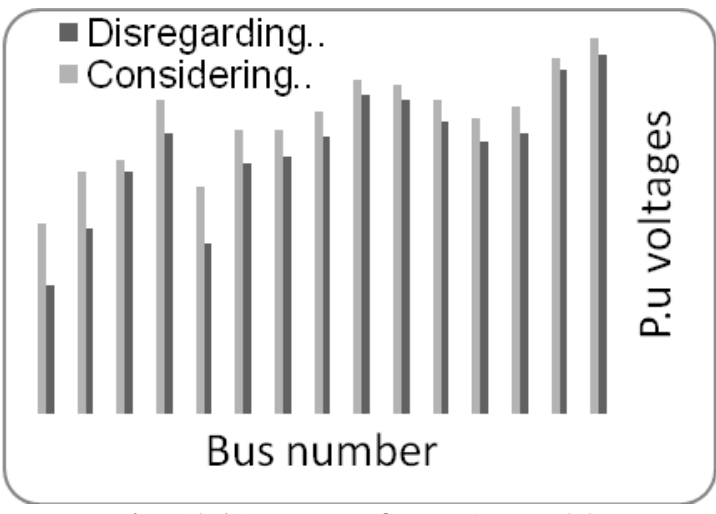

Fig (4-b) Buses from 16 to 30

Fig (4) Bus voltages when demand is reduced from $85 \%$ to 80.99.

\section{d. Heuristic calculation}

It is a trial and error method linked to the optimal power flow procedures to reach the suitable value of load reduction at every load centre. This step is required when there are violations to power-flow limits in some lines, where the limit is assumed to be $85 \%$ of the max permissible power flow. The heuristic calculations can be performed by the following steps [11]:

1- $\quad$ Optimal power flow is performed for any loading condition

2- $\quad$ The violated lines, which reach $85 \%$ of their maximum power, are defined.

3- $\quad$ The sensitivity factors " $S F_{i}$ " are defined for all buses

4- $\quad$ An initial reduction value is assumed based on the experience and the history of the process

5- $\quad$ The assumed reduction value is distributed through different buses according to their sensitivity factors.

6- The optimal power flow is performed under the new loading conditions and the violation of constraints is examined

7- $\quad$ The reduction level is modified according to the results in step 6 as follows:

- If there are lines that violate the constraints, the reduction level is increased

- If there are no lines that violate the constraints, the reduction level is decreased

8- The optimal power flow is performed under the new loading conditions and the violation of constraints is examined

9- $\quad$ Steps 7 and 8 are repeated until the critical reduction value is reached

10- If the critical value is reached, the results are tuned and the program is terminated.

Fig 5 shows the flowchart of the heuristic procedures with optimal power flow. 


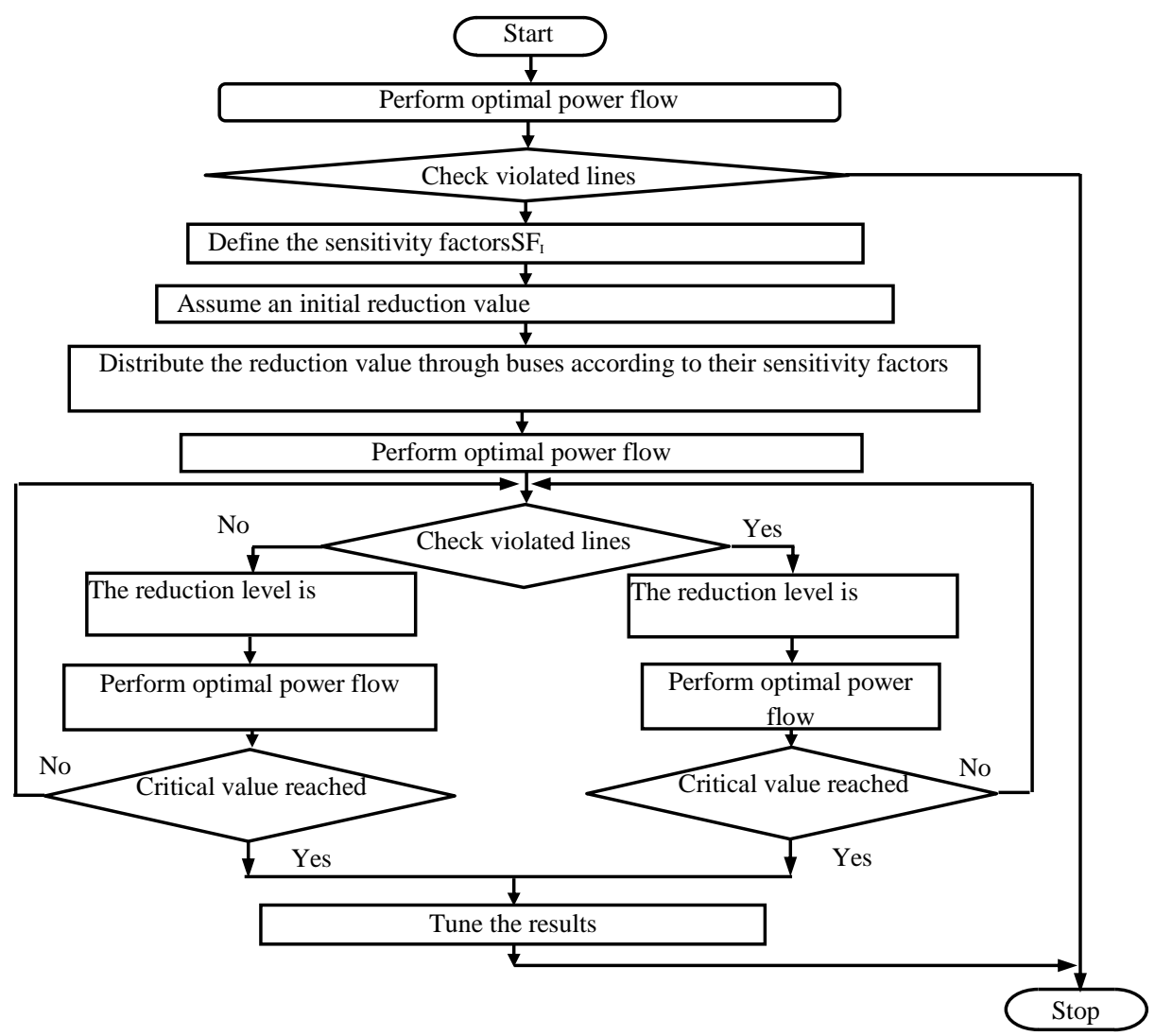

Fig (5) Flowchart of heuristic procedures with optimal power flow

\section{Heuristic Calculation Results}

Using heuristic calculations with optimal power flow program and taking into consideration the sensitivity factors, the best reduction at every load centre can be determined. Table (2) shows the best reduction for each load centre under different loading conditions. These values are calculated to overcome the increase in load demand, which can cause violation in line constraints.

The required percentage reductions at load centres decreases with the loading reduction. The required reduction values are considerably higher at buses that have more sensitive factors. 
Table (2): best reduction at different load centres

\begin{tabular}{|c|c|c|c|c|c|}
\hline \multicolumn{2}{|c|}{ For $95 \%$ loading } & \multicolumn{2}{|c|}{ For $90 \%$ loading } & \multicolumn{2}{|c|}{ For $85 \%$ loading } \\
\hline Busn umber & $\%$ red. & Bus number & $\%$ red. & Bus number & $\%$ red. \\
\hline 30 & 36.32 & 30 & 34.53 & 30 & 12.97 \\
\hline 29 & 40.00 & 29 & 40.00 & 29 & 40.00 \\
\hline 26 & 40.00 & 26 & 40.00 & 26 & 29.88 \\
\hline 24 & 40.00 & 24 & 30.29 & 24 & 9.98 \\
\hline 23 & 40.00 & 23 & 40.00 & 23 & 25.83 \\
\hline 21 & 20.91 & 21 & 11.93 & 18 & 23.35 \\
\hline 20 & 40.00 & 20 & 40.00 & 20 & 32.63 \\
\hline 19 & 31.85 & 19 & 17.86 & 21 & 4.07 \\
\hline 18 & 40.00 & 18 & 40.00 & 19 & 7.44 \\
\hline 17 & 25.84 & 17 & 12.36 & 17 & 7.18 \\
\hline 15 & 24.26 & 16 & 29.09 & 16 & 18.14 \\
\hline 16 & 40.00 & 15 & 9.78 & 15 & 7.39 \\
\hline 14 & 18.61 & 7 & 3.32 & 14 & 8.79 \\
\hline 2 & 4.93 & 14 & 11.44 & 12 & 4.11 \\
\hline 12 & 8.32 & 4 & 8.14 & 10 & 7.48 \\
\hline 3 & 29.32 & 8 & 1.95 & 7 & 1.37 \\
\hline 10 & 11.85 & 12 & 4.28 & 3 & 10.31 \\
\hline 8 & 1.89 & 10 & 6.33 & 8 & 0.79 \\
\hline 4 & 6.92 & 5 & 0.35 & 2 & 1.02 \\
\hline 7 & 2.29 & 3 & 11.49 & 5 & 0.22 \\
\hline 5 & 0.54 & 2 & 0.70 & 4 & 1.37 \\
\hline $\begin{array}{l}1,6,9,11,13 \\
22,25,27,28\end{array}$ & 0 & $\begin{array}{l}1,6,9,11,13, \\
22,25,27,28\end{array}$ & 0 & $\begin{array}{l}1,6,9,11 \\
13,22,25 \\
27,28\end{array}$ & 0 \\
\hline
\end{tabular}




\section{Load Shedding}

Load shedding can be considered as an emergency action to avoid the problems of line violation and to keep the system stable and secure [12]-[14]. Therefore, it will be the alternative of the proposed DSM technique. In other words, without DSM, the operator will be forced to follow up the load shedding methodology. Therefore, it is important to compare the situation after applying the DSM and the load shedding alternatives. The target is to study the possibility of maintaining the electricity supply using DSM, while the network is maintained operating within the acceptable limits without the need to load shedding.

Table (3) shows the percentage loading after demand reduction using DSM and using load shedding. It is clear that the final loadings after load shedding are always higher than those after demand reduction considering DSM strategy. For 95\% original loading, Table (3) shows that the differencebetween percentage demand reduction using DSM and using load shedding is approximately $2.63 \%$. This difference decreases to about $1.34 \%$ for $85 \%$ original loading. Thus, the DSM gives similar results to that of the load shedding especially with the lower original loadings. However, complete load shedding for some loads cannot be accepted as a solution for the lines violation problem. Hence, instead of complete load shedding, the demand can be managed with continuous electricity supply but the total demand reduction has to be slightly higher than that of load shedding.

Table (3) also shows that the percentages loading, which resulted after reducing the demand consideringSF, differs from one case to the other. This is attributed to the variation of the power flowing in the lines under each original loading. Thus, each case has different weight factors ( $w . f$ ) for lines and different sensitivity factors for buses. According to the sensitivity of the buses, the suitable percentage reductions are determined to solve the problem of line violation.

Table (3): Percentage loading after demand reduction using DSM and using load shedding to maintain network limits

\begin{tabular}{|c|c|c|c|}
\hline \multicolumn{1}{l|}{$\begin{array}{c}\text { Original } \\
\text { loading loading after } \\
\text { reducing the demand } \\
\text { considering (SF) }\end{array}$} & $\begin{array}{c}\text { \% loading after } \\
\text { load shedding }\end{array}$ & $\begin{array}{c}\text { \% difference in the } \\
\text { required total demand } \\
\text { reduction }\end{array}$ \\
\hline $\mathbf{9 5 \%}$ & $84.22 \%$ & $86.85 \%$ & 2.63 \\
\hline $\mathbf{9 2 \%}$ & $83.9 \%$ & $85.68 \%$ & 1.78 \\
\hline $\mathbf{9 0 \%}$ & $82.7 \%$ & $84.57 \%$ & 1.87 \\
\hline $\mathbf{8 8 \%}$ & $81.36 \%$ & $82.69 \%$ & 1.33 \\
\hline $\mathbf{8 5 \%}$ & $80.99 \%$ & $82.33 \%$ & 1.34 \\
\hline
\end{tabular}

Fig (6) and Fig(7) show the percentage power flowing in the lines of the IEEE 30-bus network for $95 \%$ and $85 \%$ loadings using DSM and load shedding. 


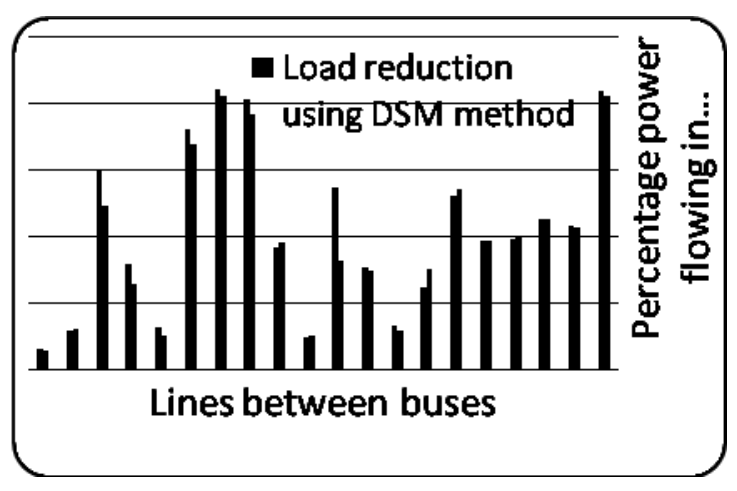

Fig (6-a): Lines from 1 to 20

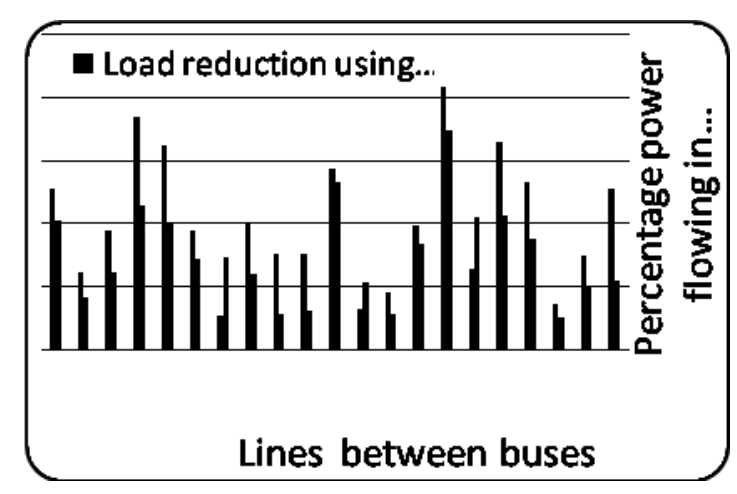

Fig (6-b): Lines from 21 to 41

Fig (6): the percentage power flowing in the lines of the IEEE 30-bus networkfor 95\% loading

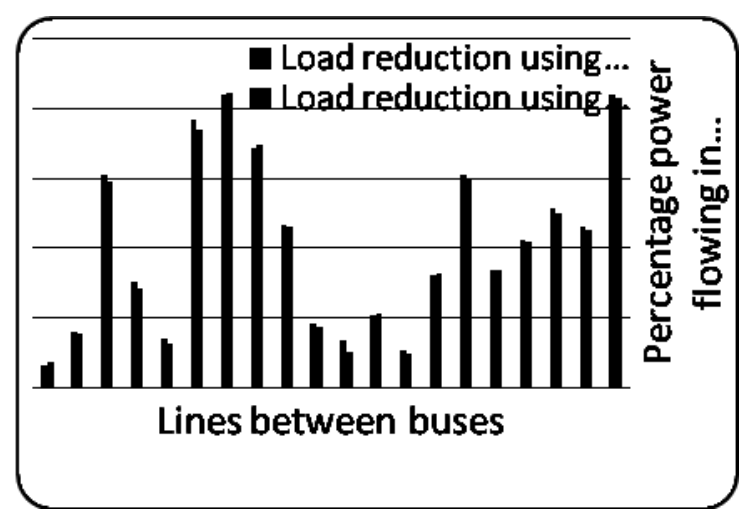

Fig (7-a): Lines from 1 to 20

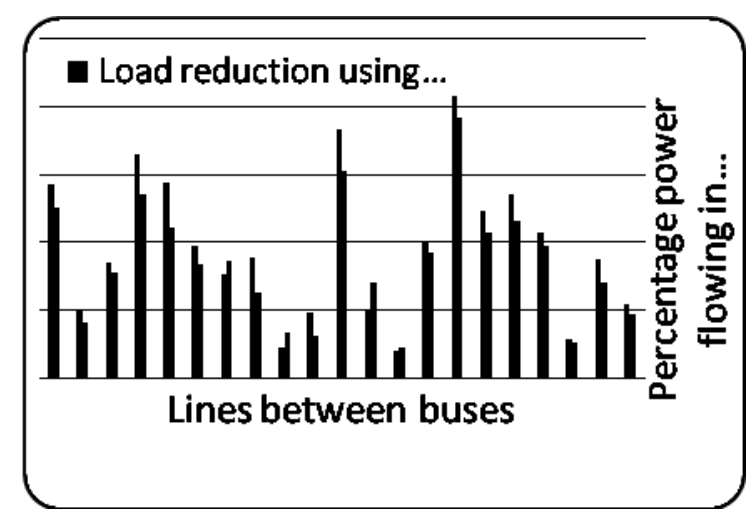

Fig (7-b): Lines from 21 to 41

Fig (7): the percentage power flowing in the lines of the IEEE 30-bus networkfor $85 \%$ loading

The use of load shedding causes higher loading for most lines compared to the use of DSM technique. For some loading conditions, e.g. $95 \%$ and $85 \%$, the number of lines that have a power exceeding $83 \%$ is greater for the case of load shedding.

Figs (8) and (9) show the voltage profile for original loadings of $95 \%$ and $85 \%$ respectively. After load reduction using the two techniques, better voltage profile is obtained for the same original loadings whenconsidering sensitivity factors. This ensures that the problem of lineconstraints violation can be solved using DSM technique considering sensitivity factors with acceptable bus voltages and continuous electricity supply. 


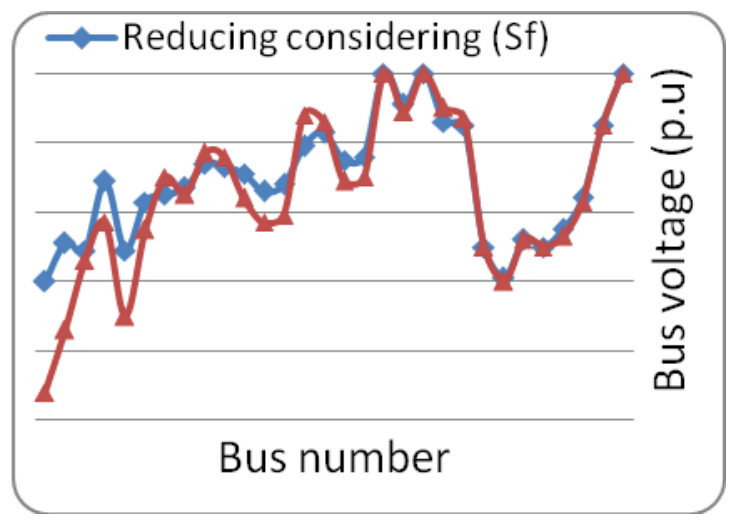

Fig (8): Voltage profile for an original loading of $95 \%$

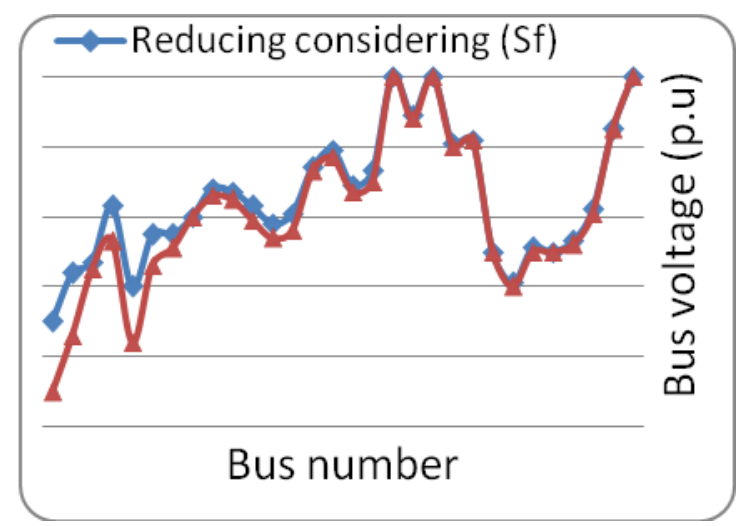

Fig (9): Voltage profile for an original loading of $85 \%$

\section{Conclusion}

In this paper, the effect of reducing the load demand using demand-side management to solve the problem of line-constraints violation has been investigated. It has been proven that the demand reduction can be more effective when sensitivity factors of load buses are taken into account. This is ensured through the observable improvement on the voltage profile, efficiency of the network and reduction of the power loss. The optimal reduction at each load centres is determined based on a heuristic algorithm taking the sensitivity factors into consideration. The required reduction level can be sent to consumers according to definite protocols based on their sensitivity factors to solve the problem of line constrains. The results ensured also that the proposed DSM technique can be followed to avoid load shedding without excessive load reduction.

\section{* References}

1- A. Molderink, V. Bakker, M.G.C. Bosman, J.L. Hurink and G. J.M. Smit, "Management and control of domestic smart grid technology", IEEE Transactions on Smart Grid, Vol. 1, No. 2, PP. 109-119, September 2010.

2- $\quad$ F. Li, W. Qiao, H. Sun, H. Wan, J. Wang, Y. Xia, Z. Xu and P. Zhang, "Smart Transmission Grid: Vision and Framework", IEEE transactions on smart grid, Vol. 1, No. 2, PP. 168-177, September2010.

3- S. Gottwalt, W. Ketter, C. Block, J. Collins and C. Weinhardt, "Demand side management- A simulation of house hold behaviour under variable prices", Applied energy, Vol. 39, No. 12, PP. 8163-8174, December 2011.

4- I. Szuvovivski , T.S.P. Fernandes and A.R. Aoki "simultaneous allocation of capacitors and voltage regulators at distribution networks using genetic algorithms and optimal power flow", 
International journal of electrical power and energy systems, Vol. 40, No. 1, PP. 62-69, September 2012.

5- M. Doostizadeh and H. Ghasemi, "A day-ahead electricity pricing model based on smart metering and demand-side management", Energy, Vol. 46, No. 1, PP. 221-230, October 2012.

6- P.P. Varaiya, F.F. Wu and J.W. Bialek, "smart operation of smart grid: risk-limiting dispatch", Proceedings of the IEEE, Vol. 99, No. 1, PP. 40-57, January 2011.

7- A.D. Giorgio and L. Pimpinella "An event driven Smart Home Controller enabling consumer economic saving and automated Demand Side Management", Applied energy, Vol. 96, PP. 92 103, August 2012.

8- $\quad$ A. Molderink, V. Bakker, M.G.C. Bosman, J.L. Hurink and G.J.M. Smit "Domestic energy management methodology for optimizing efficiency in smart grids", PowerTech, 2009 IEEE Bucharest, vol., no., pp.1,7, June 28 2009-July 22009.

9- D.Y.R. nagesh, J.V.V. Krishna and S.S. Tulasiram, "A real-time architecture for smart energy management", Innovative smart grid technologies conference, PP. 1-4, January 2010.

10- M.M. Abdullah and B. Dwolatzy, "Smart demand-side energy management based on cellular technology - A way towards smart grid technologies in Africa and low budget economies", IEEE AFRICON, 2009, AFRICON '09, vol., no., pp.1,5, 23-25 Sept. 2009

11- A. Abaza, Ahmed M. Azmy, "Demand-side management-based dynamic pricing within smart grid environment", IEEE International Conference on Smart Energy Grid Engineering (SEGE'13), 28-30 August, 2013, UOIT, Oshawa, Canada

12- J.J. Ford, H. Bevrani and G.Ledwich, "Adaptive load shedding and regional protection", Electrical power and energy systems, No. 31, PP. 611-618, 2009.

13- X. Fu and X. Wang, "Determination of load shedding to provide voltage stability", Electrical power and energy systems, No. 33, PP. 515-521, January 2011.

14- A.A. Girgis and S. Mathure, "Application of active power sensitivity to frequency and voltage variations on load sheddings", Electrical power systems research, No. 80, PP. 306-310, October 2009. 